\title{
Estudos Clínicos Iniciais com o Teste de Discriminação de Listas (TDL-UFMG)
}

\author{
Vitor Geraldi Haase ${ }^{1}$ \\ Shirley Silva Lacerda \\ Guilherme Maia de Oliveira Wood \\ Mauricio Viotti Daker \\ Marco Aurélio Lana Peixoto \\ Universidade Federal de Minas Gerais
}

\begin{abstract}
Resumo
Circuitos ligados aos lobos pré-frontais participam em uma série de funções executivas, dentre elas a organização serial do comportamento. A memória para ordem temporal é um destes aspectos e foi investigada neste estudo por meio de um teste de discriminação de listas. $\mathrm{Na}$ fase de apresentação, duas listas com dez estímulos cada (dez palavras concretas ou dez desenhos de objetos) foram apresentadas antes e depois de uma tarefa distratora. Na fase de teste foi apresentada uma lista com 20 pares de estímulos, dos quais um era novo e o outro já aparecera previamente. Os participantes precisavam reconhecer os estímulos previamente apresentados, alocando-os a cada uma das listas (julgamento de ordem temporal). Nós relatamos estudos clínicos iniciais, indicando que o procedimento pode ser útil na discriminação de adultos jovens, idosos e pacientes com patologia frontal, esclerose múltipla e pacientes psiquiátricos. Todos os grupos de pacientes, bem como o grupo de idosos, mostram uma dissociação de desempenho, exibindo reconhecimento normal e déficits nos julgamentos de ordem temporal.

Palavras-chave: Memória; episódica; recenticidade; serial; pré-frontais; executivas.
\end{abstract}

\section{Initial Clinical Investigations with the List Discrimination Test (TDL-UFMG)}

\begin{abstract}
Prefrontal cortex-related circuits are important to several executive functions, including serial organization of behavior. Serial organization of memory was investigated by means of a list discrimination task. During stimulus presentation, two lists of ten stimuli each (ten concrete word or ten line drawings of objects) were presented before and after a distracter task. In the testing phase, 20 pairs of stimuli were presented one by one. One of the stimuli in the pair was new and the other had already been presented. Participants should recognize the old stimulus and determine the list where it appeared before (temporal order judgment). We report on initial clinical investigations suggesting that the task may be useful to differentiate between young adults and older subjects, and these from patients with frontal lobe pathology, multiple sclerosis as well as psychiatric patients. All groups of patients, as well as aged subjects, showed performance dissociation with normal recognition and altered serial ordering in memory.

Keywords: Memory; episodic; prefrontal; executive.
\end{abstract}

Neste trabalho nós relatamos os resultados da avaliação clínica inicial do Teste de Discriminação de Listas (TDL-UFMG), que examina a ordem temporal na memória recente, uma habilidade relacionada às funções executivas dependentes da atividade do córtex pré-frontal. O conceito de funções executivas se desenvolveu a partir das observações, de Baddeley (1992a, 1992b), entre outros, de que a memória de curto prazo não cumpria apenas funções de armazenamento, mas também de

${ }^{1}$ Endereço para correspondência: Departamento de Psicologia, FAFICH - UFMG. Av. Antonio Carlos 6627, 31270-901 Belo Horizonte, Brasil. Fone: (31) 34996295. E-mail: haase@fafich.ufmg.br processamento de informações, ou seja, funções executivas ou de trabalho. O programa de pesquisa conduzido por Baddeley permitiu formular um modelo da memória de trabalho. Este modelo pressupõe uma divisão tripartite. Dois são sistemas escravos e suas funções se prendem ao armazenamento e recitação da informação. As propriedades destes dois sistemas, a alça fonológica e o sistema tampão viso-espacial, foram muito bem caracterizadas, inclusive o seu papel no desenvolvimento e em situações neuropatológicas (e.g., Gathercole, 1998, 1999; Torgesen, 1996). As características do executivo central permanecem bem mais obscuras. Às vezes é possível ter a impressão de que o executivo central é uma espécie de resíduo que não pode ser explicado dentro do modelo de processamento de 
informação. Ou seja, uma espécie de fantasma da máquina que vem e se comporta de modo racional, adaptativo, estratégico etc., e serve para adaptar o organismo às exigências do contexto. Isto quer dizer que a postulação da existência de um executivo central desencadeia uma regressão ao infinito. Não foge a esta regra um dos modelos mais populares do executivo central, postulado por Shallice (1988). Acreditamos que este modelo seja apenas descritivo e não explicativo, uma vez que caracteriza o funcionamento executivo em termos da dicotomia processamento automático versus processamento controlado, sem no entanto precisar a instanciação neural destes dois modos de funcionamento mental.

Por outro lado, as tentativas de caracterizar o funcionamento do executivo através de modelos mecanicistas se deparam com outro tipo de problema. Há várias propostas. Goldman-Rakic (1996), por exemplo, obteve evidências de que a região do sulco principal em primatas pode ser fraccionada de acordo com o tipo de conexão anatômica e com o tipo de informação visual processada. Já Petrides (1995) obteve evidências de que o fraccionamento pode se dar em função do tipo de processo implementado em cada região. Ocorre que, se o executivo central começar a ser fraccionado, o construto acabará se esvaziando, uma vez que a sua fragmentação terminará exigindo um mecanismo de integração que reúna os fragmentos - ou seja, um outro executivo? Vários modelos tem sido propostos para solucionar o problema da integração funcional no sistema nervoso central, o chamado binding problem (e.g., Fuster, 1995; Kelso, 1995; Pöppel, 1994; Singer, 1995). A partir de uma perspectiva dinâmica, o comportamento executivo é concebido como um emergente, o resultado de um processo construtivo que reflete as propriedades auto-organizatórias do cérebro. Mas isto tem um apelo muito mais teórico. Na prática, impõe-se a necessidade de desenvolver abordagens experimentais e psicométricas que nos permitam precisar mais detalhadamente como ocorre a integração dinâmica e contextualizada.

Do ponto de vista neuropsicológico, existe consenso a respeito de uma série de processos considerados executivos, tais como a memória de trabalho, a capacidade de inibição de estímulos interferentes e prepotentes, a capacidade de planejamento, a atitude estratégica, a consciência autonoética etc. O que não existe é um consenso sobre como hierarquizar estes construtos de alto nível e, principalmente, como comprimi-los de modo a permitir a investigação da sua instanciação neural. West (1996) realizou uma revisão sistemática e identificou as capacidades de memória de trabalho e de inibição como os denominadores comuns de uma série de modelos. Alguns autores pressupõem, entretanto, que a memória de trabalho é mais fundamental, uma vez que sem armazenamento de uma representação interna não seria possível haver inibição (e.g., Goldman-Rakic, 1987; Kimberg \& Farah, 1993). Outros pesquisadores, por sua vez, consideram que a inibição é constitutiva da memória de trabalho, porque sem inibição não seria possível proteger a informação armazenada dos estímulos interferentes e a mesma se desintegraria (e.g., Fuster, 1997).

Após uma extensa revisão da literatura, nós identificamos alguns componentes executivos que julgamos mais básicos no sentido proposto por Stuss e Benson (1986), ou seja, de que estes componentes são implementados a partir de conexões anatômicas diretas das áreas pré-frontais com porções do córtex associativo posterior e com estruturas subcorticais como a amígdala, os gânglios da base e o cerebelo. Estas interações envolvem, possivelmente, processos de sincronização oscilatória neural (e.g., Sarntheim, Petsche, Rappelsberger, Shaw \& von Stein, 1998). Os outros construtos de mais alto nível, tais como a atitude estratégica ou a consciência auto-reflexiva se derivariam então de modo construtivo, a partir de sucessivos processos de interação possibilitados pelas conexões re-entrantes entre o córtex pré-frontal e as outras áreas cerebrais. Os estudos psicométricos e experimentais do desenvolvimento da memória de trabalho, realizados por Salthouse (e.g., 1996) e outros autores (e.g., Halford, Maybery, O'Hare \& Grant, 1994; Halford, Wilson \& Phillips, 1998; Mayr, Kliegl \& Krampe, 1996) sugerem três processos: a eficiência ou velocidade de processamento, a capacidade de armazenamento temporário e a capacidade de coordenação ou complexidade relacional. A estes três devem ser acrescentados a capacidade de iniciativa e de inibição e a organização serial do comportamento. A capacidade de inibição de respostas prepotentes ou estímulos interferentes tem sido bem caracterizada nos últimos anos, tanto do posto de vista neurofisiológico quanto neurocomportamental (Dias, Robbins \& Roberts, 1997; Jonides, Smith, Marshuetz \& Koeppe, 1998; Reynolds \& Ashby, 1994), havendo inclusive evidências de que o controle inibitório e a velocidade de processamento apresentam um curso evolutivo diferenciado ao longo da vida (Williams, Ponesse, Schachar, Logan \& Tannock, 1999).

O problema da ordem serial no comportamento tem preocupado os cientistas cognitivos desde a sua formulação original por Lashley (1951). A organização serial é fundamental para uma gama de comportamentos, 
inclusive a linguagem e a própria percepção do tempo. Até há alguns anos atrás se acreditava que redes associativas jamais poderiam dar conta de implementar a ordem serial, o que tem sido consistentemente refutado tanto por modelos associativos com uma camada contextual (Burgess \& Hitch, 1999), quanto por modelos baseados em atividade oscilatória periódica (Brown, Preece \& Hulme, 2000). A questão da ordem temporal pode ser colocada, por exemplo, tanto na memória retrospectiva quanto na memória prospectiva. No que se refere à memória retrospetiva, não basta apenas recordar se um dado evento realmente aconteceu ou não. $\mathrm{Na}$ maioria das vezes, é preciso situá-lo na ordem cronológica dos eventos, recordar o que aconteceu antes, o que aconteceu depois etc. $\mathrm{O}$ mesmo argumento se aplica à memória prospectiva. A projeção para o futuro ou planejamento requer que os eventos sejam ordenados em uma seqüência temporal na memória de trabalho. Não basta, portanto, apenas reter a informação temporariamente na memória de trabalho. A informação precisa também ser organizada seqüencialmente de modo a permitir o comportamento adaptativo.

Do ponto de vista neuropsicológico, Corsi (vide Milner, 1971) desenvolveu um procedimento experimental para testar a memória de ordem temporal e mostrou que pacientes com excisões dos lobos frontais apresentavam dificuldades nesta tarefa. Nós relatamos neste artigo a nossa experiência clínica inicial com a versão de uma tarefa semelhante, simplificada para uso clínico, o Teste de Discriminação de Listas (TDL-UFMG), o qual foi adaptado a partir de procedimentos originais descritos por Butters, Kaszniak, Glisky, Eslinger e Schacter (1994) e LeFever e Kumkova (1996).

O procedimento de Corsi-Milner para o exame de recenticidade relativa emprega três tipos de estímulos: palavras, figuras de objetos e figuras abstratas (vide Milner, McAndrews \& Leonard, 1990). Na versão com palavras, por exemplo, o probando recebe uma pilha com 184 cartões. Em cada um dos cartões estão escritas duas palavras concretas, facilmente imagináveis e com uma estrutura tônica bem discriminável. $\mathrm{Na}$ forma verbal, a instrução dada é para ir lendo os nomes em voz alta e virando para o cartão seguinte. De tempos em tempos aparece um ponto de interrogação entre os dois estímulos. $O$ participante precisa então indicar qual dos dois estímulos apareceu mais recentemente. $\mathrm{Na}$ maioria das tentativas, os dois estímulos já terão aparecido anteriormente (um pode ter aparecido cinco tentativas atrás e outro, por exemplo, há 20 tentativas). Em uma tentativa controle, entretanto, um dos estímulos é novo e a tarefa se reduz a um teste de memória de Psicologia: Reflexão e Crítica, 2001, 14(2), pp. 289-304 reconhecimento. Após algumas tentativas iniciais, que constituem a base para o estabelecimento da ordem temporal, as tentativas de recenticidade e de reconhecimento passam a se alternar. $\mathrm{Na}$ versão com figuras abstratas, o número de tentativas foi reduzido para 92 e o tempo de estudo de cada figura fixado em 6 segundos.

Os estudos com a tarefa de Corsi-Milner têm indicado que o desempenho na mesma depende crucialmente de áreas do córtex pré-frontal dorsolateral (Milner, 1971; Milner, McAndrews \& Leonard, 1990, vide Petrides, 1991, para um estudo com primatas) e que pode haver uma certa especialização hemisférica, com pacientes lesionados à esquerda apresentando pior desempenho nos estímulos verbais e pacientes com lesões à direita apresentando maiores déficits relacionados aos estímulos figurais. Os achados de lateralização não tem sido, entretanto, confirmados por todos os autores (Kesner, Hopkins, \& Rineman, 1994). Considerando as evidências de que os efeitos do envelhecimento sobre a cognição parecem ser mediados por alterações estruturais nos lobos pré-frontais (West, 1996), alguns pesquisadores investigaram a possibilidade de haver um declínio na performance em função da idade, obtendo resultados confirmatórios (Cabeza, Anderson, Houle, Mangels \& Nyberg, 2000; Fabiani \& Friedman, 1997; Parkin, Walter $\&$ Hunkin, 1995). Os circuitos envolvidos na tarefa foram investigados também por métodos de neuroimagem funcional. Utilizando tomografia por emissão de pósitrons (PET), Cabeza e colaboradores (1997) observaram inicialmente que o resgate de informações relacionadas à ordem temporal ativava áreas dorsolaterais dos lobos pré-frontais, principalmente à direita; mas o circuito envolve ainda a participação de regiões posteriores (área cuneada, pré-cuneada e lobo parietal posterior direito), como é de se esperar para funções implementadas por um circuito distribuído. Um estudo de PET, mais recente realizado por Cabeza e colaboradores (2000), confirmou o padrão inicial de ativação descrito com adultos jovens, mas evidenciou que pessoas idosas apresentam comparativamente menos ativação de áreas pré-frontais à direita e mais ativação de áreas pré-frontais à esquerda. Este achado foi interpretado no sentido do recrutamento de áreas corticais adicionais, como forma de compensar os declínios cognitivos advindos do envelhecimento (vide também ReuterLorenz, Jonides, Smith, Hartley, Miller, Marshuetz \& Koeppe, 2000).

Quanto aos mecanismos envolvidos na resolução da tarefa de determinação da recenticidade relativa, existem basicamente duas hipóteses: a do processamento 
automático e a do processamento controlado. A primeira foi defendida inicialmente por Milner (e.g., Milner, McAndrews \& Leonard, 1990) e se baseia na hipótese da instanciação de um mecanismo automático de organização serial do comportamento em circuitos envolvendo os lobos pré-frontais (Hasher \& Zacks, 1979; Yntema \& Trask, 1963). Esta hipótese ganha força se considerarmos as dificuldades de processamento seqüencial observada nos pacientes com afasia de Broca (Szelag, von Steinbüchel \& Pöppel, 1997, von Steinbüchel, 1998) e nos indivíduos portadores de dislexia específica de evolução (Tallal, Merzenich, Miller \& Jenkins, 1998), os quais também apresentam alterações morfológicas nos lobos frontais, principalmente à esquerda (Galaburda \& Kemper, 1979). Os dados quanto à especialização hemisférica poderiam ser explicados de acordo com o modelo HERA (Tulving, 1998). De acordo com esta hipótese, então, as áreas dorsolaterais do lobo pré-frontal esquerdo poderiam ser responsáveis pela codificação da informação relacionada à ordem temporal, e as áreas homólogas do lobo pré-frontal direito, pelo resgate deste tipo de informação. Dados recentes de simulações com redes neurais indicam que esta é uma possibilidade viável, uma vez que tanto redes neurais conexionistas (Burgess $\&$ Hitch, 1999) quanto redes neurais baseadas em modelos de osciladores (Brown, Preece \& Hulme, 2000) exibem propriedades de organização serial da memória para eventos recentes.

A hipótese do processamento controlado na organização funcional da memória de ordem temporal tem sido defendida por diversos autores, tais como Shimamura (1995) e Mangels (1997). Segundo esta perspectiva, a ordenação temporal na memória não se origina diretamente das características do processamento neural, mas é um construto de alto nível, elaborado mentalmente a partir de representações mais simples. Um dos argumentos principais é o fato de que pacientes com excisões cirúrgicas de porções dos lobos pré-frontais apresentaram desempenho normal em uma versão da tarefa de Corsi-Milner empregando objetos reais, desde que lhes fosse permitido manipular os objetos e não apenas nomeá-los (McAndrews \& Milner, 1991). O efeito de manipulações semânticas sobre o desempenho na tarefa foi confirmado recentemente por Swain, Polkey, Bullock e Morris (1998), investigando a memória para ordem temporal em roteiros conhecidos e desconhecidos. Pacientes com ressecções cirúrgicas nos lobos frontais apresentaram déficits na seqüenciação temporal de eventos apenas para scripts desconhecidos, mas não para histórias com roteiros familiares. Estes dados parecem indicar que os déficits de performance na tarefa de recenticidade relativa podem estar relacionados à falta de controle inibitório dos estímulos interferentes, uma vez que as manipulações, que tornam as representações de ordem temporal mais salientes, tendem a facilitar ou normalizar a performance (Shimamura, 1995).

Mangels (1997) conduziu um estudo planejado especificamente para examinar a questão do processamento estratégico na memória para ordem temporal. O procedimento utilizado foi um teste de reconstrução da ordem temporal, semelhante ao que já havia sido empregado por Shimamura, Janowsky e Squire (1990). A tarefa utilizada por Shimamura e colaboradores consiste em estudar uma lista de 15 palavras, apresentadas uma a uma, e depois tentar reconstruir a ordem em que haviam sido apresentadas, manipulando uma pilha de cartões onde as palavras estavam embaralhadas. Os resultados evidenciaram que os pacientes frontais apresentaram déficits apenas na ordenação temporal de memória, enquanto pacientes amnésicos apresentavam déficits adicionais na memória de reconhecimento. Os pacientes com síndrome de Korsakoff apresentaram déficits mais severos na ordenação temporal do que outros tipos de pacientes amnésicos. No estudo de Mangels foram utilizados dois tipos de listas com 20 palavras. No primeiro tipo as palavras constituintes não eram semanticamente relacionadas, mas o eram no segundo tipo, o que permitia a sua categorização. As estratégias de aprendizagem empregadas também foram manipuladas, uma vez que em um grupo foi testada a aprendizagem incidental da ordem temporal, enquanto o outro grupo recebeu instruções explícitas indicando que sua memória seria testada. Os pacientes com lesões frontais demonstraram performance normal na ordenação temporal dos estímulos sob condições de aprendizagem incidental, indicando que a codificação da ordem serial era tão boa quanto dos controles. Ao mesmo tempo, os pacientes frontais exibiram maior efeito de interferência da manipulação semântica, ou seja, maior incidência de respostas erradas causadas por associação semântica e não por ordem temporal. Este resultado sugere que os pacientes estavam baseando suas respostas em ativações automáticas das associações semânticas, ou pelo menos, que eram mais susceptíveis à interferência destas associações irrelevantes em função de déficits no controle inibitório.

Os dados revisados acima são convincentes no sentido de indicar que as funções executivas ou de processamento controlado desempenham um papel importante na resolução da tarefa de recenticidade relativa ou na tarefa de reconstrução da ordem temporal. Mesmo assim, estamos convencidos de que a organização serial do 
comportamento é uma característica inerente ao funcionamento de redes neuronais oscilatórias, tais como o cérebro humano (Pöppel, 1978, 1994). A nossa hipótese é de que os efeitos de manipulações semânticas ou da intenção de aprender podem estar mais relacionados à dificuldade da tarefa, que é muito alta no caso de ambas as tarefas mencionadas anteriormente. Tarefas difíceis sabidamente recrutam processamento controlado e impõem demandas de memória de trabalho. Para investigar esta questão mais a fundo, consideramos que se fazia necessário utilizar uma tarefa mais simples, como por exemplo, o procedimento de discriminação de listas (Butters, Kaszniak, Glisky, Eslinger \& Schacter, 1994, LeFever \& Kumkova, 1996). A tarefa de discriminação de listas envolve a apresentação dos estímulos em duas listas diferentes, marcadas por algum estímulo saliente ou tarefa distratora. $\mathrm{Na}$ fase de teste, o probando deve indicar então em qual lista apareceu o estímulo. A tarefa de discriminação de listas pode ser interpretada como um teste de determinação da ordem temporal na memória e exibe sensibilidade para alterações relacionadas a lesões nos lobos frontais e ao envelhecimento (Moscovitch \& Winocur, 1995). Nós adaptamos então um Teste de Discriminação de Listas (TDL-UFMG) para uso com a população brasileira. Atualmente estamos empregando apenas um procedimento de aprendizagem incidental, na tentativa de verificar como o desempenho evolui ao longo do curso da vida e quais são as possibilidades de utilização clínica. Este trabalho relata a nossa experiência clínica inicial com o TDL-UFMG. Mais especificamente, o trabalho objetiva verificar se o procedimento pode ter utilidade clínica na diferenciação do desempenho de pacientes com lesões ou disfunções dos lobos frontais e, ao mesmo tempo, verificar se a tarefa pode também ser de utilidade na avaliação dos efeitos do processo normal de envelhecimento sobre as funções mnemônicas.

\section{Método}

\section{Participantes}

A amostra investigada se constituiu de 99 participantes, sendo que 69 constituíram o grupo controle e 30, o grupo clínico. Todos os indivíduos participaram voluntariamente no estudo após esclarecimento dos seus objetivos e obtenção do consentimento informado. A Tabela 1 descreve os dados demográficos da amostra. Os integrantes do grupo controle foram recrutados entre estudantes universitários $(n=58$, idade média $=23,72$ anos, $d p=5,93)$ e entre participantes idosos de um programa

Psicologia: Reflexão e Crítica, 2001, 14(2), pp. 289-304 de treinamento cognitivo para seniores $(n=11$, idade média $=65,62, d p=8,61)$. Os participantes do grupo controle foram testados nas dependências do Centro de Aplicação da Psicologia (CEAP-UFMG). As pessoas do grupo clínico são pacientes do Hospital das Clínicas da UFMG e o estudo foi realizado no âmbito da uma avaliação neuropsicológica mais abrangente, realizada como parte de sua investigação clínica. O grupo clínico foi subdividido em pacientes psiquiátricos $(n=14$, idade média $=31,86, d p=11,39$ ), pacientes com esclerose múltipla ( $n=11$, idade média $=42,55, d p=7,89)$, pacientes com síndrome frontal $(n=5$, idade média $=55,40$, $d p=16,49)$. Os grupos não foram pareados quanto às características demográficas. Os diagnósticos do grupo clínico, no caso dos pacientes psiquiátricos, foram realizados de acordo com os critérios do DSM-IV. Onze pacientes apresentaram quadros de esquizofrenia, dois pacientes, quadros depressivos e um paciente foi diagnosticado como portador de transtorno afetivo bipolar. Todos os pacientes psiquiátricos se encontravam em fase de remissão dos seus sintomas. Os pacientes com esclerose múltipla participam de um programa longitudinal de tratamento farmacológico e de acompanhamento neuropsicológico. Todos os pacientes com esclerose múltipla apresentam diagnósticos definitivos da doença, conforme evidências clínicas, laboratoriais, neuroradiológicas, neurofisiológicas e de evolução do curso clínico (conforme critérios de Poser e cols., 1983). Os portadores de esclerose múltipla foram testados também em períodos de remissão clínica da doença. $\mathrm{O}$ grupo de pacientes com disfunções neurológicas frontais é muito heterogêneo e reduzido. Deste modo, estes pacientes foram excluídos de algumas análises estatísticas. No grupo com patologia frontal, as evidências neuropsicológicas apontavam para comprometimentos hemisféricos bilaterais e foram realizados os seguintes diagnósticos: seqüela de hemorragia espontânea intraparenquimatosa, transtorno de personalidade após traumatismo fechado de crânio, neurossífilis terciária do tipo paralisia geral progressiva, quadro inicial de demência frontal e seqüela de meningioma. Foram selecionados para compor a amostra todos os pacientes atendidos pela equipe no segundo semestre de 1999 e no primeiro semestre de 2000, que correspondessem às categorias diagnósticas definidas e que demonstrassem compreender o funcionamento da tarefa de memória para ordem temporal. Desta forma, foram excluídos aqueles casos exibindo comprometimento cognitivo mais severo e mais abrangente. 
Tabela 1. Caracterização da Amostra

\begin{tabular}{|c|c|c|c|c|c|c|c|c|}
\hline Grupo & Descrição & $n$ & & Idade & & & Escolaridade & Sexo $(\%$ Fem $)$ \\
\hline & & & Min & $\operatorname{Max}$ & Média & $d p$ & & \\
\hline 1 & Adultos Jovens & 58 & 12 & 45 & 23,72 & 5,93 & $2^{\circ}$ Compl. & 48,3 \\
\hline 2 & Idosos & 11 & 56 & 85 & 65,62 & 8,61 & $2^{\circ}$ Incompl. & 84,6 \\
\hline 3 & Psiquiátricos & 14 & 15 & 51 & 31,86 & 11,39 & $2^{\circ}$ Incompl. & 50,0 \\
\hline 4 & Esclerose Múltipla & 11 & 28 & 59 & 42,55 & 7,89 & $3^{\circ}$ Incompl. & 84,6 \\
\hline 5 & Síndrome Frontal & 5 & 28 & 68 & 55,40 & 16,49 & $2^{\circ}$ Incompl. & 40,0 \\
\hline
\end{tabular}

\section{Instrumento}

O Teste de Discriminação de Listas tem como objetivo a avaliação da memória de recenticidade e de reconhecimento e foi adaptado por Lacerda e Haase (2000) a partir de uma simplificação clínica do procedimento de discriminação de listas proposta inicialmente por LeFever e Kumkova (1996). O procedimento consiste de dois tipos de tarefa (reconhecimento e recenticidade), apresentados em duas modalidades de estímulos (pictoriais e verbais). $\mathrm{Na}$ fase de apresentação dos estímulos é solicitado ao participante que nomeie (ou leia) uma série de vinte estímulos pictoriais (ou verbais, na lista alternativa) que vão sendo mostrados pelo examinador à razão de um estímulo a cada 3 ou 4 segundos (vide Figura 1). Após a nomeação (ou leitura) dos dez primeiros estímulos, é pedido ao participante apresentação dos estímulos inicia-se a fase de testagem, em que são apresentados ao probando vinte pares de figuras (ou de palavras), sendo que, em cada par, um dos estímulos foi apresentado anteriormente e o outro é um estímulo novo. $\mathrm{Na}$ fase de testagem, o participante deve indicar, dentre os dois estímulos, qual já foi apresentado anteriormente e, a seguir, se este mesmo estímulo apareceu antes ou depois da tarefa interveniente de contagem regressiva.

Os estímulos utilizados são exibidos nas Tabelas $2 \mathrm{e}$ 3. Os estímulos verbais foram selecionados com base em um conjunto de critérios. Todos os estímulos são: a) substantivos concretos, b) de fácil representação pela imaginação visual, c) de alta freqüência no vocabulário, inclusive de crianças, d) com uma estrutura fonológica bem nítida (palavras espondaicas). Os itens verbais foram

Lista de Estímulos

Lista A

JANELA

ESCADA

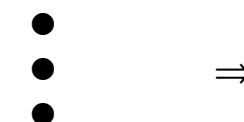

PORTA

PIPOCA

Lista de Teste

ESPADA CORDA

COELHO
SALADA

TDL-UFMG: Estímulos Verbais

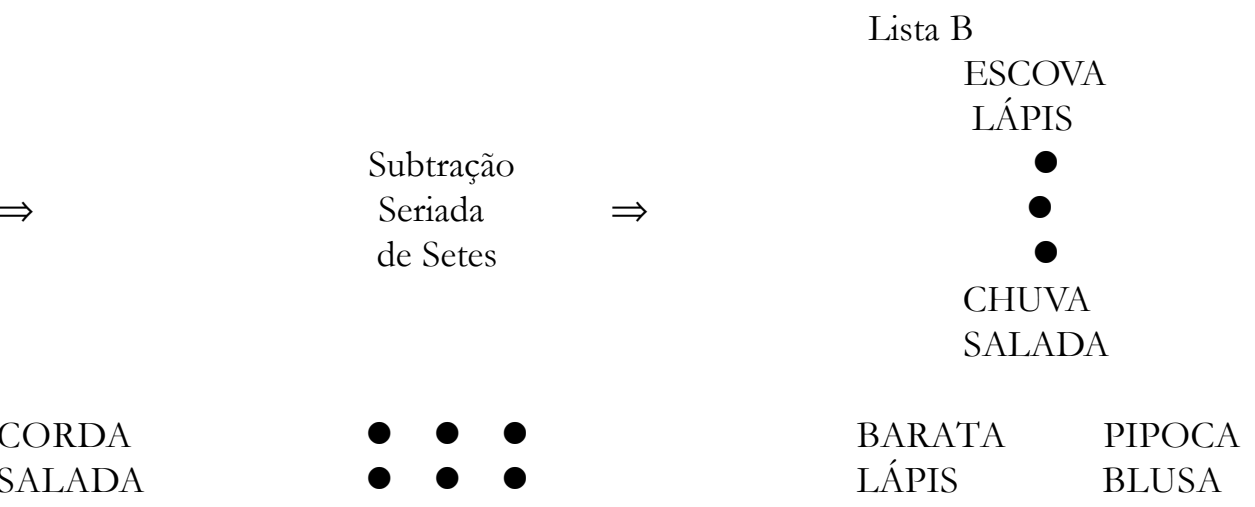

Figura 1. Procedimento de aplicação do TDL-UFMG

que realize uma prova de subtração seriada de 100-7 durante 60 segundos (contagem regressiva em voz alta e de 7 em 7, a partir de 100). Esta contagem regressiva, além de funcionar como um marcador temporal, tem a função de distrair o sujeito e limpar o sistema tampão da memória de trabalho. Segue-se a nomeação (ou leitura) dos dez estímulos restantes. Logo após esta fase de escolhidos com base no inventário de freqüência de palavras em livros didáticos infantis coligido por Pinheiro (1996). Para a seleção dos estímulos pictoriais foi utilizado um catálogo de figuras publicado por Canongia (1981) e que é de utilização corrente no tratamento fonoaudiológico em nosso meio. As figuras consistem de contornos ou traçados de linha representando objetos 
da vida cotidiana. Os nomes das figuras selecionadas para compor as listas pictoriais satisfazem também os critérios para a construção das listas verbais. Adicionalmente, a adequação das figuras foi checada, tendo como juizes diversos estudantes de graduação e pós-graduação em psicologia.
Teoricamente, o delineamento do teste permite a diferenciação entre distúrbios dos lobos temporais mediais, que já devem comprometer a performance no reconhecimento e comprometimentos frontais, que devem comprometer a performance apenas no que se refere à determinação da ordem temporal de apresentação dos

Tabela 2. Estímulos Pictoriais Utilizados no TDL-UFMG

\begin{tabular}{lll}
\hline Nomeação & \multicolumn{2}{c}{ Teste } \\
\hline Casa & Dado & Torneira \\
Tesoura & Barraca & Serrote \\
Carro & Cinto & Escada \\
Balde & Cadeira & Sacola \\
Faca & Casa & Panela \\
Cavalo & Pêra & Faca \\
Banana & Banana & Copo \\
Fogão & Pena & Tesoura \\
Picolé & Mala & Gravata \\
Serrote & Revólver & Vela \\
Chapéu & Pincel & Chapéu \\
Cadeira & Balde & Violão \\
Bola & Chave & Cabide \\
Escada & Vestido & Carro \\
Óculos & Bola & Folha \\
Chave & Cigarro & Óculos \\
Dado & Bolo & Lâmpada \\
Mala & Cavalo & Castelo \\
Lâmpada & Sapo & Fogão \\
Revólver & Picolé & Cama \\
\hline
\end{tabular}

Tabela 3. Estímulos Verbais Utilizados no TDL-UFMG

\begin{tabular}{lll}
\hline Nomeação & \multicolumn{2}{c}{ Teste } \\
\hline Boca & Sapato & Bandeira \\
Garrafa & Livro & Garrafa \\
Porco & Macaco & Banco \\
Dente & Caneta & Pipoca \\
Cabelo & Gravata & Chuva \\
Flores & Porta & Sorvete \\
Macaco & Tijolo & Dente \\
Alface & Lápis & Blusa \\
Pipoca & Barata & Janela \\
Forno & Porco & Fogueira \\
Jarra & Caderno & Jarra \\
Salada & Cabelo & Pedra \\
Chuva & Forno & Bolsa \\
Lápis & Coelho & Salada \\
Escova & Barbante & Boca \\
Porta & Espada & Corda \\
Sapato & Fósforo & Alface \\
Espada & Sino & Colher \\
Janela & Mamão & Flores \\
Sino & Escova & Rede \\
\hline
\end{tabular}


estímulos. A questão da determinação da lateralidade da lesão tem suscitado resultados contraditórios. Uma possível lateralização à direita para os déficits nos estímulos pictoriais não foi confirmada por todos os autores (Kesner, Hopkins, \& Rineman, 1994). Isto é compreensível, dados os resultados indicando a preservação das capacidades de nomeação de estímulos no caso de funcionamento isolado do hemisfério direito (Mohr, Pulvermüller \& Zaidel, 1994; Pulvermüller, 1996). Dados iniciais indicam que a tarefa de reconhecimento é relativamente fácil, havendo efeito de teto a partir dos 6 anos de idade, e que o teste como um todo pode ser empregado com crianças a partir dos 4 anos de idade (Lacerda \& Haase, 2000). Dados não publicados, obtidos em nosso laboratório, indicam que, em uma amostra de crianças em idade escolar, os coeficientes de fidedignidade situam-se ao redor de 0,9 para o teste de reconhecimento e ao redor de 0,6, para os testes de recenticidade. Os coeficientes de fidedignidade para testes de memória episódica, na maioria das vezes, não ultrapassam esta barreira de 0,6, podendo, portanto, ser considerados aceitáveis (e.g., American Academy of Neurology, 1996; Lezak, 1995; Spreen \& Strauss, 1998).

\section{Procedimento}

O procedimento empregado foi de aprendizagem incidental, ou seja, durante a fase de apresentação dos estímulos, os participantes não eram avisados de que sua memória seria testada. Apenas eram solicitados a nomear ou ler os estímulos. Os participantes foram testados individualmente em uma sala bem iluminada e silenciosa. Primeiramente foi realizada a testagem com a lista de estímulos pictoriais e depois com a lista de estímulos verbais. $\mathrm{Na}$ avaliação clínica, foi tomado o cuidado de não testar as duas listas durante a mesma sessão, para evitar interferência. No caso da coleta de dados com participantes-controle isto não foi possível. A aplicação individual consiste na apresentação manual dos 40 cartões de cada uma das listas, verbal ou pictorial. A aplicação é dividida em duas fases: apresentação e teste. $\mathrm{Na}$ fase de apresentação, o participante deve nomear ou ler os estímulos. O intervalo entre os estímulos é de cerca de 3 a 4 segundos. Após a nomeação ou leitura do $10^{\circ}$ estímulo, começa a contagem regressiva de sete em sete a partir de 100, por 60 segundos, a qual também é realizada em voz alta. Logo depois da contagem, o sujeito reinicia a nomeação a partir do $11^{\circ}$ estímulo até o $20^{\circ}$. Tanto nesta fase como na seguinte o examinador anota, na folha apropriada, as respostas dadas pelo sujeito. Assim que termina a fase de apresentação inicia-se a fase de teste, em que cada par de estímulos é apresentado por 10 segundos, incluindo o tempo para as perguntas, e o intervalo entre os estímulos será de 3 segundos. $\mathrm{Na}$ fase de teste, é utilizado um procedimento de escolha forçada, em que o probando deve responder a duas perguntas: "Qual destas figuras (palavras) você viu anteriormente?" e "Esta figura (palavra) apareceu antes ou depois da contagem?"

Quatro escores são computados, correspondendo às percentagens de acertos com relação a cada uma das perguntas e para as duas modalidades de estimulação: reconhecimento pictorial, reconhecimento verbal, recenticidade pictorial e recenticidade verbal. No cômputo dos escores de recenticidade é considerada a percentagem em relação ao escore de acertos na modalidade reconhecimento. Assim, por exemplo, se o participante acertou 17 itens em uma modalidade de reconhecimento e 13 itens na modalidade equivalente de recenticidade, os seus escores percentuais equivalerão a $85(17 / 20)$ para o reconhecimento e 76,5 (13/17). A probabilidade teórica de acerto caso o indivíduo escolha aleatoriamente todas as respostas é de 50\%.

Tabela 4. Distribuição dos Escores Percentuais no TDL-UFMG

\begin{tabular}{|c|c|c|c|c|c|c|c|}
\hline & $\begin{array}{c}\text { Adultos } \\
\text { Jovens }\end{array}$ & Idosos & Psiquiátricos & $\begin{array}{c}\text { Esclerose } \\
\text { Múltipla }\end{array}$ & $\begin{array}{c}\text { Síndrome } \\
\text { Frontal }\end{array}$ & $\begin{array}{c}\text { Controle } \\
\text { (1 e } 2)\end{array}$ & $\begin{array}{l}\text { Clínico } \\
(3,4 \text { e } 5)\end{array}$ \\
\hline & $\begin{array}{l}\text { Média } \\
\text { (dp) }\end{array}$ & $\begin{array}{l}\text { Média } \\
\text { (dp) }\end{array}$ & $\begin{array}{l}\text { Média } \\
\text { (dp) }\end{array}$ & $\begin{array}{c}\text { Média } \\
\text { (dp) }\end{array}$ & $\begin{array}{c}\text { Média } \\
(d p)\end{array}$ & $\begin{array}{c}\text { Média } \\
(d p)\end{array}$ & $\begin{array}{c}\text { Média } \\
\text { (dp) }\end{array}$ \\
\hline Reconh. & 97,58 & 98,63 & 94,64 & 97,27 & 100,00 & 97,75 & 96,61 \\
\hline Pictorial & $(3,65)$ & $(3,23)$ & $(13,22)$ & $(4,67)$ & $(0,00)$ & $(3,59)$ & $(9,34)$ \\
\hline Reconh. & 95,60 & 88,63 & 91,07 & 88,50 & 82,00 & 94,49 & 88,33 \\
\hline Verbal & $(5,22)$ & $(7,77)$ & $(11,46)$ & $(17,48)$ & $(18,57)$ & $(6,19)$ & $(14,64)$ \\
\hline Recent. & 89,57 & 67,46 & 62,29 & 72,47 & 55,00 & 86,05 & 65,25 \\
\hline Pictorial & $(8,52)$ & $(17,34)$ & $(13,38)$ & $(14,40)$ & $(20,41)$ & $(13,10)$ & $(15,61)$ \\
\hline Recent. & 84,99 & 69,47 & 62,92 & 66,22 & 38,25 & 82,52 & 60,48 \\
\hline Verbal & $(12,55)$ & $(13,98)$ & $(12,51)$ & $(14,53)$ & $(10,29)$ & $(13,91)$ & $(15,79)$ \\
\hline
\end{tabular}




\section{Resultados}

Os procedimentos de testagem foram bem aceitos por todos os participantes, revelando-se de aplicação rápida e fácil. É importante ressaltar também que a aplicação dos procedimentos não é entediante ou estafante, nem tampouco suscita dificuldades muito acima da capacidade cognitiva dos pacientes e idosos. As distribuições dos escores para os diversos grupos estudados são exibidas na Tabela 4.

Conforme esperado, as diferenças entre os diversos grupos quanto à performance no teste de reconhecimento variaram muito pouco. Comparando os grupos controle com a totalidade do grupo clínico, é possivel observar que as performances situaram-se na faixa dos $95 \%$ para o reconhecimento pictorial (médias de 97,75 e de 96,61 para o grupo controle e clínico, respectivamente, $t=1,281$, $g l=90, p<0,204)$ e $90 \%$ para o reconhecimento verbal (médias de 94,49 e e de 88,33,61 para o grupo controle e clínico, respectivamente, $t=2,205, g l=90, p<0,030$ ), havendo diferenças significativas entre os dois grupos apenas no que se refere ao reconhecimento verbal. Uma análise univariada de variância, tendo os escores nos testes como variável dependente e o grupo como fator, revelou a ausência de efeito principal $(F=1,097, g l=92, p<0,354)$ e de diferenças entre os grupos para a memória de reconhecimento pictorial no teste de Scheffe. Já no que se refere à memória de reconhecimento verbal, a análise de variância revelou um efeito principal significativo $(F=3,795, g l=92, p<0,0134)$, mas não foi observada nenhuma diferença significativa entre os diversos subgrupos no teste de Scheffe (adultos jovens vs. idosos $v s$. pacientes de esclerose múltipla vs. pacientes psiquiátricos). A observação dos dados da Tabela 1 permite verificar ainda que, de um modo geral, o desempenho nos testes com estímulos pictoriais foi superior aos testes verbais para todas as modalidades de testagem. Estas análises sugerem que o efeito principal observado no caso do reconhecimento verbal poderia estar ligado a um déficit mais geral de performance com estímulos verbais no grupo clínico. Esta impressão é corroborada se considerarmos que as médias de cada um dos grupos situaram-se ao redor de um ponto de corte de $95 \%$ para o reconhecimento pictorial e de $85 \%$ para o reconhecimento verbal (com exceção do grupo de pacientes frontais, em que a média foi igual a $82 \%$ ). É possível afirmar, portanto, com segurança, que o desempenho dos diversos subgrupos que compõem o grupo clínico nas tarefas de reconhecimento ultrapassou com folga os níveis determinados pelo acaso.

A performance nas tarefas de determinação da ordem temporal de apresentação dos estímulos revelou, por outro lado, diferenças significativas entre os grupos. A pior performance foi exibida, como era esperado, pelos pacientes com quadros neuropsicológicos lesionais clássicos de comprometimento pré-frontal. A performance deste grupo nas tarefas de recenticidade situou-se em uma faixa média de variação ao redor de $50 \%$ ou abaixo. O grupo frontal foi excluído das análises de variância em função do pequeno número de participantes e da heterogeneidade das etiopatogenias. Duas análises univariadas de variância foram então realizadas, tendo os subgrupos etiológicos como fator e o desempenho em recenticidade pictorial e verbal, respectivamente, como variável dependente. Para a recenticidade pictorial foi observado tanto um efeito principal significativo $(F=30,332, g l=92, p<0,00001)$ quanto diferenças significativas entre os grupos no teste de Scheffe. As principais diferenças observadas foram entre os grupos de adultos jovens e pacientes psiquiátricos (diferença média $=27,29, p<0,000$ ), entre adultos jovens e idosos (diferença média $=22,12, p<0,0001$ ) e entre adultos jovens e pacientes de esclerose múltipla (diferença média $=17,10, p<0,0001)$. O desempenho dos portadores de esclerose múltipla foi superior ao dos idosos (diferença média $=5,01, p<0,782)$ e o destes superior ao dos pacientes psiquiátricos (diferença média $=517, p<0,743$ ), mas as diferenças entre os grupos não foram significativas. A análise post hoc evidenciou, portanto, a formação de dois subconjuntos quanto ao desempenho, um formado pelos adultos jovens (média $=89,57, d p=8,52$ ) e outro formado pelos pacientes psiquiátricos (média $=62,29$, $d p=13,38$ ), idosos (média $=67,46, d p=17,34$ ) e portadores de esclerose múltipla (média $=72,47, d p=14,40$ ). A existência deste padrão de diferenciação entre os grupos foi confirmada por múltiplos testes $t$ de Student (Tabela 5). De um modo generalizado, é possível afirmar que as médias de performance em recenticidade pictorial situaram-se ao redor de $90 \%$ para os adultos jovens e $70 \%$ para os demais grupos, com exceção do grupo exibindo patologia frontal, cuja performance foi de ordem aleatória, constituindo um terceiro patamar.

A análise das diferenças de performance na tarefa de recenticidade verbal foi globalmente significativa $(F=15,893, g l=92, p<0,000)$ e permitiu também a constituição de três patamares de performance. $\mathrm{O}$ primeiro grupo é formado pelos pacientes frontais com performance aleatória no teste (da ordem de 40\%), a seguir vem o grupo de pacientes psiquiátricos, idosos e portadores de esclerose múltipla com médias de performance situadas entre 60 e $70 \%$, e, finalmente os adultos jovens com performance equivalente a cerca de $85 \%$ de acertos. Este agrupamento post-hoc da performance em recenticidade verbal foi permitido por uma análise de variância, mostrando a presença tanto de um efeito 
principal significativo $(F=15,90, g l=92, p<0,0001)$ quanto pelos testes de Scheffe. O teste de Scheffe evidenciou um padrão que discrimina o desempenho em recenticidade verbal entre os grupos adultos jovens e pacientes psiquiátricos (diferença média $=22,07, p<0,000$ ), adultos jovens e pacientes de esclerose múltipla (diferença média $=18,77, p<0,000$ ) e entre adultos jovens e idosos (diferença média $=15,52, p<0,006$ ). Novamente, não houve diferenças quanto ao desempenho dos grupos de pacientes psiquiátricos e pacientes de esclerose múltipla (diferença média $=3,30, p<0,946$ ), e entre ambos estes grupos de pacientes e os idosos (diferença média $=6,56$, $p<0,676$ e diferença média $=3,26, p<0,954$, para as difernças com pacientes psiquiátricos e de esclerose múltipla, respectivamente).

Considerando que a distribuição dos escores para a amostra como um todo não era normal, tal como determinada pelo teste de Kolmogorov-Smirnoff e com exceção do caso da recenticidade verbal, resolvemos checar os resultados por meio da realização de testes nãoparamétricos. O emprego de procedimentos nãoparamétricos nos permitiu estender as análises para o grupo de pacientes com quadros frontais clássicos. A realização de múltiplos testes de Kruskal-Wallis permitiu confirmar a presença de um efeito principal para as diferenças entre os grupos quanto aos escores em recenticidade verbal $\left(x^{2}=44,58, p<0,0001\right)$, recenticidade pictorial $\left(x^{2}=44,20, p<0,0001\right)$ e reconhecimento verbal $\left(x^{2}=15,06, p=0,006\right)$, mas não quanto ao reconhecimento pictorial $\left(x^{2}=3,49, p=0,479\right)$. De um modo geral, o padrão de diferenças entre os grupos, obtidos com a realização de múltiplos testes de Mann-Whitney confirmou o padrão estabelecido pela análise de variância e pelos testes $\mathrm{T}$ (vide Tabela 6). Os testes de Mann-Whitney permitiram,

Tabela 5. Diferenças de Desempenho entre os Grupos (Teste $t$ )

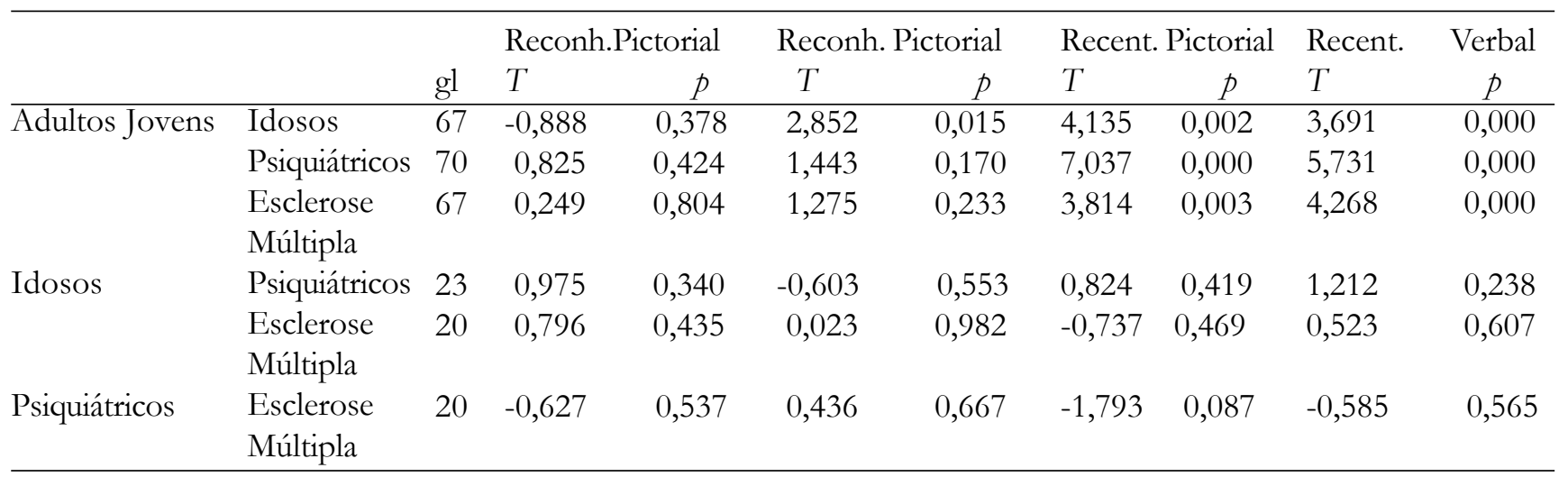

Tabela 6. Diferenças de Desempenho entre os Grupos (Teste de Mann-Whitney)

\begin{tabular}{|c|c|c|c|c|c|c|c|c|c|}
\hline & & \multicolumn{2}{|c|}{ Reconh. Pictorial } & \multicolumn{2}{|c|}{ Reconh. Verbal } & \multicolumn{2}{|c|}{ Recent.Pictorial } & \multicolumn{2}{|c|}{ Recent. Verbal } \\
\hline & & $U$ & $p$ & $U$ & $p$ & $U$ & $p$ & & $p$ \\
\hline \multirow[t]{5}{*}{ Adultos Jovens } & Idosos & 265,50 & 0,291 & 153,50 & 0,004 & 82,50 & 0,000 & 120,00 & 0,001 \\
\hline & Psiquiátricos & 399,50 & 0,913 & 293,00 & 0,088 & 45,00 & 0,000 & 53,00 & 0,000 \\
\hline & Esclerose & 311,50 & 0,884 & 195,50 & 0,082 & 101,50 & 0,000 & 69,50 & 0,000 \\
\hline & Múltipla & & & & & & & & \\
\hline & Síndrome Frontal & 92,50 & 0,188 & 51,00 & 0,014 & 19,50 & 0,002 & 3,00 & 0,000 \\
\hline \multirow[t]{3}{*}{ Idosos } & Psiquiátricos & 63,50 & 0,467 & 54,00 & 0,222 & 58,50 & 0,459 & 57,00 & 0,424 \\
\hline & Esclerose & 53,50 & 0,652 & 40,00 & 0,314 & 49,50 & 0,478 & 51,50 & 0,809 \\
\hline & Múltipla & & & & & & & & \\
\hline \multirow[t]{3}{*}{ Psiquiátricos } & Síndrome Frontal & 22,50 & 0,583 & 25,00 & 0,827 & 13,50 & 0,280 & 1,00 & 0,003 \\
\hline & Esclerose & 73,50 & 0,851 & 65,00 & 0,796 & 41,50 & 0,082 & 51,00 & 0,410 \\
\hline & Múltipla & & & & & & & & \\
\hline Esclerose & Síndrome Frontal & 22,50 & 0,257 & 20,00 & 0,186 & 15,00 & 0,245 & 6,00 & 0,023 \\
\hline Múltipla & Síndrome frontal & 20,00 & 0,441 & 16,00 & 0,310 & 10,50 & 0,138 & 3,00 & 0,014 \\
\hline
\end{tabular}


por outro lado, evidenciar um padrão mais claro de diferenciações entre os grupos no que se refere ao desempenho em reconhecimento verbal. $O$ que se observa na Tabela 6 é que as únicas diferenças inter-grupos significativas para o reconhecimento verbal dizem respeito, respectivamente, às discriminações entre adultos jovens e idosos $(U=153,50, p=0,004)$ e adultos jovens e pacientes com distúrbios frontais $(U=51,00, p=0,014)$. Ora, o que os dois grupos de idosos (idade média $=65,62, d p=8,61$ ) e pacientes com síndrome frontal (idade média $=55,40$, $d p=16,49)$ tem em comum é a idade mais avançada (vide Tabela 1). Desta forma, as diferenças observadas, e não esperadas, quanto à performance em reconhecimento verbal podem ser provisoriamente atribuídas à idade.

A aplicação das técnicas não-paramétricas permitiu ainda incluir o grupo de pacientes com patologias frontais nas análises. $O$ padrão evidenciado na Tabela 6 mostra claramente que, de um modo geral, os grupos não diferem quanto ao desempenho em reconhecimento e que o desempenho nos testes de recenticidade se distribui por três patamares. O patamar mais alto de performance é ocupado pelos jovens universitários, o segundo patamar pelas pessoas idosas, pelos portadores de esclerose múltipla e pelos pacientes psiquiátricos e, o último patamar, pelos pacientes frontais. As análises confirmam então uma dissociação de performance entre a memória de reconhecimento e a memória para ordem temporal, tanto para os pacientes com síndrome frontal, em que o desempenho em ordem temporal é aleatório, quanto para os outros dois grupos clínicos e para os idosos, cujo desempenho é significativamente comprometido, mas não de modo tão severo.

Considerando a possibilidade de que a juventude e a maior escolarização do grupo de universitários esteja contribuindo para a sua melhor performance, realizamos outras análises, em que os dois grupos de controle (de jovens e idosos) foram agrupados e comparados como um todo com os grupos clínicos. Tanto os dados de testes T, quanto os dados do teste de Mann-Whitney, evidenciaram um claro padrão de dissociação entre os desempenhos em memória de reconhecimento e de recenticidade. Houve diferenças de desempenho entre os grupos controle e clínico no que se refere à recenticidade verbal $(T=5,63, p<0,0001, U=235,00$, $p<0,0001)$, à recenticidade pictorial $(T=5,42, p<0,0001$, $\mathrm{U}=309,50, p<0,0001)$ e ao reconhecimento verbal ( $T=2,21, p=0,030, U=736,50, p=0,018)$, mas não houve diferenças quanto à performance em reconhecimento pictorial ( $T=1,28, p=0,204, U=1020,00, p=0 ., 651)$.

Uma estimativa inicial da validade psicométrica preditiva do TDL-UFMG pode ser obtida a partir do

Psicologia: Reflexão e Crítica, 2001, 14(2), pp. 289-304 cálculo dos coeficientes de correlação de Spearman entre os diversos escores e o grupo (controle ou clínico) a que os participantes pertenciam. Os resultados evidenciaram correlações significativas entre o grupo e os escores em recenticidade verbal $(r=0,583, p<0,0001)$, recenticidade pictorial $(r=0,535, p<0,0001)$ e reconhecimento verbal $(r=0,236, p=0,007)$, mas não para o reconhecimento pictorial $(r=0,046, p=0,653)$. As correlações entre os escores do TDL-UFMG e as variáveis demográficas sexo e nível de escolaridade não foram significativas.

A validade preditiva dos procedimentos com o TDLUFMG foi examinada também por meio de uma análise de regressão logística (cf. Bühl \& Zöffel, 1999). Inicialmente, foram conduzidas análises isoladas para cada um dos escores, tendo o grupo (controle ou clínico) como variável dependente e cada uma das tarefas do TDLUFMG como variável independente. Como pode ser visto pelos valores de $x^{2}$ e respectivos níveis de significância exibidos na Tabela 7, os modelos de regressão logística não foram significativos apenas para o escore em reconhecimento pictorial. As análises isoladas de regressão logística resultaram em equações com parâmetros estatisticamente significativos apenas para o desempenho nos testes de recenticidade, mas não para os testes de reconhecimento (vide Tabela 7). Como havia 69 indivíduos no grupo controle e 30 no grupo clínico a chance de acertar a aleatoriamente a classificação para o grupo de maior freqüência é da ordem de $70 \%$. A inspeção das percentagens totais de classificações corretas permitidas pelos modelos na Tabela 7 indica que as mesmas são da ordem de $70 \%$ para os escores de reconhecimento. Enquanto isto, a percentagem total de classificações corretas para os escores em recenticidade verbal é igual a $77,1 \%$, sendo igual a $79,4 \%$ para a recenticidade pictorial. Desta forma, o modelo matemático não acrescenta nenhuma precisão às classificações feitas a partir dos escores isolados em reconhecimento e melhora em 7 a 9,5\% as classificações efetuadas com os escores isolados em recenticidade. Os dados da Tabela 7 indicam também que a especificidade dos testes isolados de recenticidade na amostra estudada é da ordem de 0,93 a 0,91, o que é bastante razoável. Enquanto isto, a sensibilidade, para os testes isolados, é bastante baixa, variando entre 0,37 e 0,50. A baixa sensibilidade pode ser atribuída ao pequeno número de pacientes portadores de comprometimentos frontais clássicos. Ao mesmo tempo, os comprometimentos dos pacientes com esclerose múltipla e até mesmo dos pacientes psiquiátricos nas tarefas de ordem temporal não são de intensidade muito severa. 
Tabela 7. Resultados e Classificações Possibilitadas pelas Análises Isoladas de Regressão Logística (expressas em percentagens)

\begin{tabular}{lcccccccccccc}
\hline Tarefa & $\begin{array}{c}\text { Total de } \\
\text { Classificações } \\
\text { Corretas }\end{array}$ & $\begin{array}{c}F \\
\text { Neg }\end{array}$ & $\begin{array}{c}F \\
\text { Pos }\end{array}$ & $\begin{array}{c}V \\
\text { Pos }\end{array}$ & $\begin{array}{c}V \\
\text { Neg }\end{array}$ & $\begin{array}{c}\text { Qui- } \\
\text { Quadr, }\end{array}$ & Sig & $b$ & $a$ & Wald & Sig \\
\hline $\begin{array}{l}\text { Reco } \\
\text { Pict } \\
\text { Reco }\end{array}$ & 71,4 & 96,6 & 0,0 & 3,4 & 100,0 & 0,989 & 0,320 & 0,035 & 2,541 & 0,930 & 0,335 \\
$\begin{array}{l}\text { Verb } \\
\text { Recent }\end{array}$ & 74,2 & 89,3 & 0,0 & 10,7 & 100,0 & 6,350 & 0,012 & 0,059 & 4,526 & 4,874 & 0,027 \\
$\begin{array}{l}\text { Pict } \\
\text { Recent }\end{array}$ & 79,4 & 50,0 & 8,7 & 50,0 & 91,3 & 31,781 & 0,000 & 0,085 & 5,655 & 22,082 & 0,000 \\
Verb & 77,1 & 63,0 & 7,2 & 37,0 & 92,8 & 33,314 & 0,000 & 0,093 & 5,884 & 19,146 & 0,000 \\
\hline
\end{tabular}

Legenda: F Neg: Falsos Negativos, F Pos: Falso Positivos, V Pos: Verdeiros Positivos, V Neg:

Verdadeiros Negativos.

A seguir foi calculada uma equação-ônibus de regressão logística, tendo a pertinência de grupo como variável dependente e todos os escores do TDL como variáveis independentes. No rol de variáveis independentes foram incluídas também as variáveis demográficas sexo, idade e nível de escolaridade. O teste global de significância do modelo evidenciou $x^{2}=49,202$ e $p<0,0001$. Já o teste de Hosmer e Lemeshow serviu para rejeitar a hipótese nula $\left(x^{2}=2,511, p=0,961\right)$. Os coeficientes de Wald para cada uma das variáveis dependentes são exibidos na Tabela 8. As únicas variáveis independentes que geraram equações significativas foram os dois escores de recenticidade, com coeficiente de Wald igual a 11,503 $(p=0,001)$ para a recenticidade verbal e igual a 6,850 $(p=0,009)$ para a recenticidade pictorial. Os parâmetros para os escores em reconhecimento e para as variáveis verbal e Wald $=6,850, p=0,009$ para a recenticidade pictorial). Os resultados da equação-ônibus de regressão logística permitiram classificar corretamente um total de $84,2 \%$ dos casos (vide Tabela 9), obtendo um ganho de precisão nas classificações igual a 14\% acima do nível do acaso. A especificidade da classificação com base em todas as variáveis independentes foi igual a 0,928, permanecendo no mesmo patamar atingido pelas variáveis isoladas, enquanto a sensibilidade aumentou para 0,615 .

Com o intuito de estabelecer pontos de corte provisórios que orientassem a utilização clínica da TDLUFMG a partir dos dados atualmente disponíveis, examinamos as freqüências de distribuição dos escores nas amostras de controles e de pacientes (vide Tabelas 10 e 11). Uma estimativa mais conservadora, com o intuito

Tabela 8. Resultados da Análise - Ônibus de Regressão Logística

\begin{tabular}{lcrl}
\hline & $b$ & Wald & Sig. \\
\hline Reconhecimento Pictorial &,- 095 & 2,595 & 0,107 \\
Recenticidade Pictorial &,- 064 & 6,850 & 0,009 \\
Reconhecimento Verbal &, 050 & 1,847 & 0,174 \\
Recenticidade Verbal &,- 107 & 11,503 & 0,001 \\
Sexo & 1,137 & 2,240 & 0,134 \\
Idade &,- 022 & 1,020 & 0,312 \\
Grau de Instrução &, 383 & 3,494 & 0,062 \\
a & 14,823 & 6,667 & 0,010 \\
\hline
\end{tabular}

demográficas não atingiram níveis estatísticos de significância, com exceção de uma tendência par ao nível instrução (Wald=3,494, $p=0,062$ ). Já os parâmetros das equações para as tarefas de reconhecimento foram todos significativos (Wald $=11,503, p=0,001$ para a recenticidade de garantir a especificidade, pode ser obtida estabelecendo os pontos de corte no percentil 10 da amostra controle. Enquanto isto, uma estimativa mais liberal, visando ao aumento da sensibilidade, é estabelecida quanto o corte é efetuado no percentil 25. A partir destes procedimentos, 
Tabela 9. Classificações Possibilitadas pela Análise - Ônibus de Regressão Logística (expressas em percentagens)

\begin{tabular}{ccccc}
\hline Total de & $F$ & $F$ & $V$ & $V$ \\
Classificações Corretas & Neg & Pos & Pos & Neg \\
\hline 84,2 & 38,5 & 7,2 & 61,5 & 92,8 \\
\hline
\end{tabular}

Legenda: F Neg: Falsos Negativos,F Pos: Falso Positivos, V Pos: Verdadeiros Positivos, V Neg: Verdadeiros Negativos.

foi possível estabelecer, provisoriamente, as seguintes faixas de variação para as diversas tarefas. O desempenho normal na tarefa de reconhecimento pictorial provavelmente se situa acima dos escores 90 a 95, sendo 80 e 85 os valores equivalentes para o teste de reconhecimento verbal. No que se refere aos testes de recenticidade, os pontos de corte devem oscilar entre 70 e 80 para a modalidade pictorial e entre 65 e 75 para ordem temporal com estímulos verbais. Estes resultados correspondem às médias de acertos de 70 e $80 \%$ para as tarefas de recenticidade, observadas respectivamente nas amostras de LeFever e Kumkova (1996) e Butters, Kaszniak, Glisky, Eslinger e Schacter (1994). É importante frisar, entretanto, que estas são apenas diretrizes iniciais.

\section{Discussão}

A hipótese inicial quanto aos resultados é de que haveria diferenças significativas entre os grupos controle e grupo clínico apenas quanto ao desempenho em

Tabela 10. Diretrizes Iniciais para o Estabelecimento de Pontos de Corte (distribuição dos escores no grupo controle)

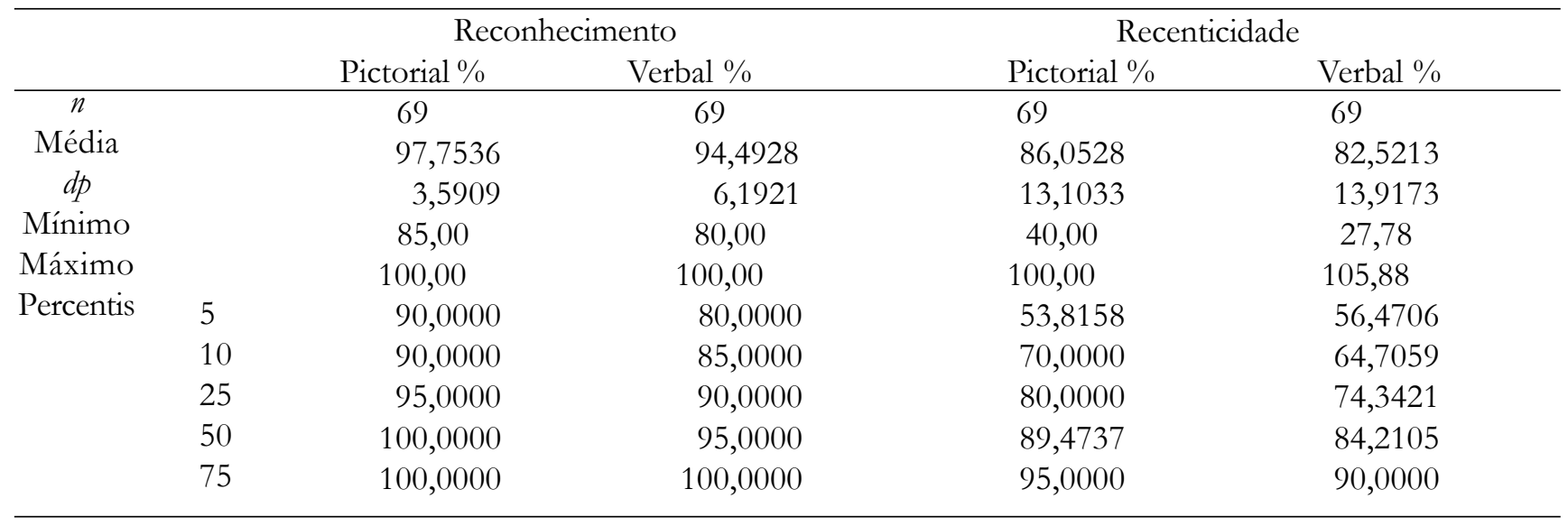

Tabela 11. Diretrizes Iniciais para o Estabelecimento de Pontos de Corte (distribuição dos escores no grupo clínico)

\begin{tabular}{|c|c|c|c|c|c|}
\hline & & \multicolumn{2}{|c|}{ Reconhecimento } & \multicolumn{2}{|c|}{ Recenticidade } \\
\hline & & Pictorial \% & Verbal \% & Pictorial \% & Verbal $\%$ \\
\hline$n$ & & 30 & 30 & 30 & 30 \\
\hline Média & & 96,6129 & 88,3333 & 65,2517 & 60,4898 \\
\hline$d p$ & & 9,3441 & 14,6413 & 15,6135 & 15,7905 \\
\hline Mínimo & & 50,00 & 40,00 & 40,00 & 23,53 \\
\hline Máximo & & 100,00 & 100,00 & 90,00 & 83,33 \\
\hline Percentis & 5 & 74,0000 & 45,5000 & 42,0000 & 27,4510 \\
\hline & 10 & 90,0000 & 57,5000 & 44,9444 & 36,1404 \\
\hline & 25 & 95,0000 & 85,0000 & 50,6579 & 47,3684 \\
\hline & 50 & 100,0000 & 95,0000 & 64,9123 & 64,7059 \\
\hline & 75 & 100,0000 & 95,0000 & 83,7500 & 73,6842 \\
\hline
\end{tabular}


memória para ordem temporal, mas não para a memória de reconhecimento. Da mesma forma, era esperado que o desempenho dos idosos fosse significativamente mais baixo que o dos adultos jovens também apenas no que se refere à memória de ordem temporal. A princípio, não existem quaisquer razões para supor que os grupos clínicos ou de idosos possam apresentar déficits na memória de reconhecimento. De outra parte, existem diversas evidências indicando que tanto idosos (West, 1996), quanto pacientes com esclerose múltipla (Arnett, Rao, Grafman, Berardin, Luchetta, Binder \& Lobeck, 1997) e esquizofrenia (Clark \& O'Carroll, 1998; Cohen, Braves \& O’Reilly, 1996), apresentam déficits de desempenho relacionados às funções cognitivas e de memória que envolvem circuitos frontais e subcorticais. É importante ressaltar, entretanto, o caráter provisório dos resultados aqui descritos. As amostras são ainda muito pequenas e de tamanhos diferentes, e até o momento não foi possível obter um bom controle das variáveis demográficas, tais como idade, sexo e nível de escolarização formal. Mesmo assim, os resultados são animadores, principalmente se considerarmos as diferenciações entre os grupos controle e clínico como um todo, situação em que foi possível obter um melhor controle das variáveis demográficas.

Os resultados indicam que o Teste de Discriminação de Listas (TDL-UFMG) é adequado para a utilização com a população brasileira adulta, que é sensível às diferenças causadas pelo envelhecimento e que o mesmo consegue discriminar um grupo controle de um grupo clínico com disfunções diversas dos lobos pré-frontais e de circuitos relacionados. As hipóteses iniciais foram confirmadas, na medida em que as diferenças de performance em memória de reconhecimento entre os diversos grupos, - com exceção do reconhecimento verbal dos grupos com idade mais avançada - não foram significativas, ao passo que a performance em recenticidade discriminou os grupos controle dos grupos clínicos. Principalmente a análise de regressão logística contribuiu para confirmar a existência de uma dissociação na performance e que a diferenciação entre os grupos é possibilitada pelos testes de recenticidade, mas não pelos testes de reconhecimento. A dissociação de performance é bem mais nítida para os pacientes frontais, mas também evidente nos outros dois grupos clínicos e de idosos. Déficits semelhantes na memória para ordem temporal já haviam sido relatados em indivíduos idosos (Fabiani \& Friedman, 1997), em pacientes com esclerose múltipla (Beatty \& Monson, 1991) e em pacientes com Parkinson (Gabrieli, 1996). Tanto quanto é do nosso conhecimento, este é o primeiro relato evidenciando déficits na memória para ordem temporal em pacientes psiquiátricos, principalmente esquizofrênicos.

Quanto ao debate sobre o tipo de processamento informacional engajado pela resolução de tarefas de julgamento de ordem temporal (Mangels, 1997), os nossos resultados não são definitivos no sentido de excluir a participação de processamento controlado. A observação, entretanto, de que uma tarefa bem mais simples, envolvendo apenas aprendizagem incidental, consegue discriminar a performance de grupos clínicos com comprometimentos frontais bastante sutis, é prova de que a dificuldade da tarefa é um fator a ser considerado.

$\mathrm{O}$ fato de que os procedimentos de testagem da memória de reconhecimento não se revelaram adequados para discriminar entre os grupos, não significa que eles devam ser abandonados. Ao contrário, no presente contexto, a testagem da memória de reconhecimento cumpre a importante função de demonstrar a dissociação de performance. É bom lembrar, que na avaliação neuropsicológica não importa apenas em documentar a presença de déficits. A constatação de funções preservadas é igualmente importante. Para documentar a utilidade do teste de reconhecimento haveria a necessidade de investigar uma população de pacientes com amnésia e/ ou lesões do lobo temporal, tal como doentes de Alzheimer. É bom frisar também, que no presente estudo foram excluídos todos os pacientes com graus tão severo de comprometimento que não conseguissem compreender o funcionamento da tarefa de recenticidade.

O exame de algumas características psicométricas das tarefas mostrou que a sua especificidade é bastante razoável, enquanto a sensibilidade ainda é baixa. Possivelmente, a inclusão de mais pacientes com quadros típicos de alterações frontais na amostra deve aumentar a sensibilidade. Os resultados disponíveis até o momento, permitem, entretanto, afirmar que uma performance aleatória (50-60\%), nos testes de recenticidade, é claramente indicativa de comprometimento frontal, enquanto performances intermediárias, na faixa de 70 a $80 \%$ são suspeitas. Os desempenhos intermediários são sugestivos de comprometimento de circuitos envolvendo os lobos frontais em pacientes com esquizofrenia ou esclerose múltipla. Mas evidentemente, ninguém está sugerindo utilizar o TDL-UFMG para o diagnóstico clínico destas duas entidades. Enquanto isto, com base no desempenho da amostra atual, performances abaixo de $90 \%$ nos testes de reconhecimento devem ser indicativas de patologia dos lobos temporais. Esta afirmação deve ser examinada, investigando o desempenho de pacientes com amnésia ou outros tipos de pacientes com patologias envolvendo os lobos temporais, tais como portadores de doença de Alzheimer ou epilepsia.

Psicologia: Reflexão e Crítica, 2001, 14(2), pp. 289-304 


\section{Referências}

American Academy of Neurology (1996). Assessment: Neuropsychological testing of adults. Considerations for neurologists. Neurology, 47, 592-9.

Arnett, P. A., Rao, S. M., Grafman, J., Berardin, L., Luchetta, T., Binder, J. R. \& Lobeck, L. (1997). Executive functions in multiple sclerosis: An analysis of temporal ordering, semantic encoding, and planning abilities. Neuropsychology, 11, 535-544.

Baddeley, A. (1992a). Is working memory working? Quarterly Journal of Experimental Psychology, 44A, 1-31.

Baddeley, A. (1992b). Working memory. Science, 255, 556-559.

Beatty, W. W. \& Monson, N. (1991). Memory for temporal order in multiple sclerosis. Bulletin of the Psychonomic Society, 29, 10-12.

Brown, G. D. A., Preece, T. \& Hulme, C. (2000). Oscillator-based memory for serial order. Psychological Revien, 107, 127-181.

Burgess, N. \& Hitch, G. J. (1999). Memory for serial order: A network model of the phonological loop and its timing. Psychological Review, 106, 551-581.

Bühl, A. \& Zöffel, P. (1999). SPSS version 8. Einfübrung in die moderne Datenanalyse unter Windows. München: Addison-Wesley.

Butters, M. A., Kaszniak, A. W., Glisky, E. L., Eslinger, P. J. \& Schacter, D. L. (1994). Recency discrimination deficits in frontal lobe patients. Neuropsychology, 8, 343-353.

Cabeza, R., Mangels, J. A., Nyberg, L., Habib, R., Houle, S., McIntosh, A. R. \& Tulving, E. (1997). Brain regions differentially involved in remembering what and when: A PET study. Neuron, 19, 663-670.

Cabeza, R., Anderson, N. D., Houle, S., Mangels, J. \& Nyberg, L. (2000). Agerelated differences in neural activity during item and temporal-order memory retrieval: A positron emission tomography study. Journal of Cognitive Neuroscience, 12, 197-206.

Canongia, M.B. (1981). Técnica de Aplicação de Figuras para Evocação. Rio de Janeiro: Atheneu. São Paulo.

Clark, O. \& O'Carroll, R. E. (1998). An examination of the relationship between executive function, memory, and rehabilitation status in schizophrenia. Neuropsychological Rehabilitation, 8, 229-241.

Cohen, J. D., Braves, T. S. \& O’Reilly, R. C. (1996). A computational approach to prefrontal cortex, cognitive control and schizophrenia: recent developments and current challenges. Philosophical Transactions of the Royal Society of London B, 351, 1515-1527.

Dias, R., Robbins, T. W. \& Roberts, A. C. (1997). Dissociable forms of inhibitory control within prefrontal cortex with an analog of the Wisconsin Card Sorting Test: Restriction to novel situations and independence from "on line" processing. Journal of Neuroscience, 17, 9285-9297.

Elman, J. L. (1995). Language as a dynamical system. Em R. F. Port \& T. van Gelder (Orgs.), Mind as motion. Explorations in the dynamics of cognition (pp. 195-226). Cambridge, MA: MIT Press.

Fabiani, M. \& Friedman, D. (1997). Dissociations between memory for temporal order and recognition memory in aging. Neuropsychologia, 35, 129-141.

Fuster, J. M. (1995). Memory in the cerebral cortex. An empirical approach to neural networks in the buman and nonbuman primate. Cambridge: MIT Press.

Fuster, J. M. (1997). The prefrontal cortex. anatomy, physiology, and neuropsychology of the frontal lobe ( $3^{\text {a }}$. ed.). Philadelphia/New York: Lippincot/Raven.

Gabrieli, J. D. E. (1996). Memory systems analyses of mnemonic disorders in aging and age-related diseases. Proceedings of the National Academy of Sciences (USA), 93, 13534-13540.

Galaburda, A. M. \& Kemper, T. L. (1979). Cytoarchitectonic abnormalities in developmental dyslexia: A case study. Annals of Neurology, 6, 94-100.

Gathercole, S.E. (1988). The development of memory. Journal of Child Psychology and Psychiatry, 39, 3-27.
Gathercole, S. E. (1999). Cognitive approaches to the development of short-term memory. Trends in Cognitive Sciences, 3, 410-419.

Goldman-Rakic, P. S. (1987). Circuitry of primate prefrontal cortex and regulation of behavior by representational memory. Em V. B. Mountcastles, F. Plum \& S.R. Geiger (Orgs.), Handbook of physiology. Section I: The nervous system. Vol. V: Higher functions of the brain. Part I (pp. 373-417). Bethesda: American Physiological Society.

Goldman-Rakic, P.S. (1996). Regional and cellular fractionation of working memory. Proceedings of the National Academy of Sciences (USA), 93, 1347313480.

Halford, G. S., Maybery, M., O’Hare, T. \& Grant, P. (1994). The development of memory and processing capacity. Child Development, 65, 1338-1356.

Halford, G. S., Wilson, W. H. \& Phillips S. (1998). Processing capacity defined by relational complexity: Implications for comparative, developmental, and cognitive psychology. Behavioral and Brain Sciences, 21, 803-831.

Hasher, L. \& Zacks, R. T. (1979). Automatic and effortful processes in memory. Journal of Experimental Psychology: General, 106, 356-388.

Janowsksy, J. S., Shimamura, A. P. \& Squire, L. R. (1989). Source memory impairment in patients with frontal lobe lesions. Neuropsychologia, 27, 1043-1056.

Johnson, M. K. (1997). Source monitoring and memory distortion., Philosophical Transactions of the Royal Society of London B, 352, 1733-1745.

Jonides, J., Smith, E. E., Marshuetz, C. \& Koeppe, R. A. (1998). Inhibition in verbal working memory revealed by brain activation. Proceedings of the National Academy of Sciences (USA), 95, 8410-8413.

Kelso, J. A. S. (1995). Dynamic patterns: The self-organization of brain and behavior. Cambridge: MIT Press.

Kesner, R. P., Hopkins, R. O. \& Rineman, B. (1994). Item and order dissociation in humans with prefrontal cortex damage. Neuropsychologia, 32, 881-891.

Kimberg, D. Y. \& Farah, M. J. (1993). A unified account of cognitive impairments following frontal lobe damage: The role of working memory in complex, organized behavior. Journal of Experimental Psychology: General, 4, 411-428.

Lacerda, S. S. \& Haase, V. G. (2000). Exame neuropsicológico da memória de reconhecimento e da memória de recenticidade. Em V.G. Haase, R. Rothe-Neves, C. Käppler, M. L. M. Teodoro \& G. M. O. Wood (Orgs.), Psicologia do desenvolvimento: Contribuições interdisciplinares (pp. 161178). Belo Horizonte: Health.

Lashley, K. S. (1951). The problem of serial order in behavior. Em L.A. Jeffress (Org.), Cerebral mechanisms in behavior.The Hixon symposium (pp. 112-146). New York/London: Wiley/Chapman \& Hall.

LeFever, F. F. \& Kumkova, E. I. (1996). The recency test: clinical adaptation of an experimental procedure for studying frontal lobe function with implications for lateral specialization and different modes of temporal judgment. Brain and Cognition, 30, 286-289.

Lezak, M. D. (1995). Neuropsychological assessment (3a ed.). New York: Oxford University Press.

Mangels, J. A. (1997). Strategic processing and memory for temporal order in patients with frontal lobe lesions. Neuropsychology, 11, 207-221.

Mayr, U., Kliegl, R. \& Krampe, R. T. (1996). Sequential and coordinative processing dynamics in figural transformations across the life span. Cognition, 59, 61-90.

McAndrews, M. P. \& Milner, B. (1991). The frontal cortex and memory for temporal order. Neuropsychologia, 29, 849-859.

Milner, B. (1971) Interhemispheric differences in the localization of psychological processes in man. British Medical Bulletin, 27, 272-277.

Milner, B., McAndrews, M. P. \& Leonard, G. (1990). Frontal lobes and memory for the temporal order of recent events. Cold Spring Harbor Symposia on Quantitative Biology, 55, 987-994.

Mohr, B., Pulvermüller, F. \& Zaidel, E (1994). Lexical decision after left, right and bilateral presentation of function words, content words and non-words: Evidence for interhemispheric interaction. Neuropsychologia, 32, 105-124. 
Parkin, A. J., Walter, B. M. \& Hunkin, M. (1995). Relationships between normal aging, frontal-lobe function, and memory for temporal and spatial information. Neuropsychology, 9, 304-312.

Moscovitch, M. \& Winocur, G. (1995). Frontal lobes, memory, and aging. Annals of the New York Academy of Sciences, 769, 119-150.

Petrides, M. (1991). Functional specialization within the dorsolateral frontal cortex for serial order memory. Proceedings of the Royal Society of London B, 246, 299-306.

Petrides, M. (1995). Functional organization of the human frontal cortex for mnemonic processing. Annals of the New York Academy of Sciences, 769, 85-96.

Pinheiro, A. M. V. (1996). Contagem de freqüencia de ocorrência de palavras expostas a crianças na faixa pré-escolar e séries iniciais do $1^{\circ}$ grau. São Paulo: Associação Brasileira de Dislexia.

Poser, C. M., Paty, D. W., Scheinberg, L., McDonald, W. I., Davis, F. A., Ebers, G. C., Johnson, K. P., Sibley, W. A., Silberberg, D. H. \& Tourtellotte, W. W. (1983). New diagnostic criteria for multiple sclerosis: guidelines for research protocols. Annals of Neurology, 13, 227-231.

Pöppel, E. (1978). Time perception. Em R. Held, H. W. Leibowitz \& H. L. Teuber (Orgs.), Handbook of sensory physiology. Vol. 8: Perception (pp. 713729). Berlim: Springer.

Pöppel, E. (1994). Temporal mechanisms in perception. International Review of Neurobiology, 37, 185-202.

Pulvermüller, F. (1996). Hebb's concept of cell assemblies and the psychophysiology of word processing. Psychophysiology, 33, 317-333.

Reuter-Lorenz, P. A., Jonides, J., Smith, E. E., Hartley, A., Miller, A., Marshuetz, C. \& Koeppe, R. A. (2000). Age differences in the frontal lateralization of verbal and spatial working memory revealed by PET. Journal of Cognitive Neuroscience, 12, 174-187.

Reynolds, C. \& Ashby, P. (1999). Inhibition in the human motor cortex is reduced just before a voluntary contraction. Neurology, 53, 73-735.

Salthouse, T. A. (1996). The processing-speed theory of adult age differences in cognition. Psychological Bulletin, 103, 403-428.

Sarntheim, J., Petsche, H., Rappelsberger, P., Shaw, G. L. \& von Stein, A. (1998). Synchronization between prefrontal and posterior association cortex during human working memory. Proceedings of the National Academy of Sciences (USA), 95, 7092-7096.

Shallice, T. (1988). Information-processing models of consciousness: possibilities and problems. Em A.J. Marcel \& E. Bisiach (Orgs.), Consciousness in contemporary science (pp. 305-333). Oxford: Clarendon Press.

Shima, K., Mushiake, H.l Saito, N. \& Tanji, J. (1996). Role for cells in the presupplementary motor area in updating motor plans. Proceedings of the National Academy of Sciences (USA), 93, 8694-8698.

Shimamura, A. P. (1995). Memory and frontal lobe function. Em Gazzaniga, M.S. (Org.), The cognitive neurosciences (pp. 803-814). Cambridge: MIT Press.
Shimamura, A. P., Janowksy, J. S. \& Squire, L. S. (1990). Memory for the temporal order of events in patients with frontal lobe lesions and amnesic patients. Neuropsychologia, 28, 803-813.

Singer, W. (1995). Time as a coding space in neocortical processing: A hypothesis. Em M.S. Gazzaniga (Org.), The cognitive neurosciences (pp. 91104). Cambridge: MIT.

Singer, W. (1995). Consciousness and the structure of neuronal representations. Philosophical Transactions of the Royal Society of London B, 353, 1829-1840.

Spreen, O. \& Strauss, E. (1998). A compendium of neuropsychological tests. Administration, norms, and commentary. New York: Oxford University Press.

Stuss, D. T. \& Benson, D. F. (1986). The frontal lobes. New York: Raven.

Swain, A. A., Polkey, C. E., Bullock, P. \& Morris, R. G. (1998). Recognition memory and memory for order in script-based stories following frontal lobe excisions. Cortex, 34, 25-45.

Szelag, E., von Steinbüchel, N. \& Pöppel, E. (1997). Temporal processing disorders in patients with Broca's aphasia. Neuroscience Letters, 10, 33-36.

Tallal, P., Merzenich, M. M., Miller, S. \& Jenkins, W. (1998). Language learning impairments: Integrating basic science, technology, and remediation. Experimental Brain Research, 123, 210-219.

Torgesen, J. K. (1996). A model of memory from an information processing perspective: The special case of phonological memory. Em G. R. Lyon \& N. A. Krasnegor (Orgs.), Attention, memory, and executive function (pp. 157-184). Baltimore: Paul H. Brookes.

Tulving, E. (1998). Brain/mind correlates of human memory. Em M. Sabourin, F. Craik \& M. Robert (Orgs.), Advances in psychological sciences. Vol. 2: Biological and cognitive aspects (pp. 441-460). Hove, UK: Psychology Press.

von Steinbüchel, N. (1998). Temporal ranges of central nervous processing: Clinical evidence. Experimental Brain Research, 123, 220-233.

Weiss, P., Stelmach, G. E. \& Hefter, H. (1997) Programming of a movement sequence in Parkinson's disease. Brain, 120, 91-102.

West, R.L. (1996). An application of prefrontal cortex function theory to cognitive aging. Psychological Bulletin, 120, 272-292.

Williams, B. R., Ponesse, J. S., Schachar, R. J., Logan, G. D. \& Tannock, R. (1999). Development of inhibitory control across the life span. Developmental Psychology, 35, 205-213.

Yntema, D. \& Trask, F. (1963). Recall as a search process. Journal of Verbal Learning and Verbal Behavior, 2, 65-74.

\section{Sobre os autores:}

Vitor Geraldi Haase é Médico Neurologista, Professor Adjunto no Departamento de Psicologia da UFMG, coordenador do Laboratório de Neuropsicologia do Desenvolvimento da UFMG, mestre em lingüistica pela PUCRS e Doutor em Biologia Humana pela Universidade de Munique.

Shirley Silva Lacerda é Psicólogo pela UFMG. Bolsista de aperfeiçoamento no Laboratório de Neuropsicologia do Desenvolvimento da UFMG.

Guilherme Maia de Oliveira Wood é Psicólogo e Mestre em Psicologia Social pela UFMG, Doutorando em Neuropsicologia pela Universidade de Aachen.

Maurício Viotti Daker é Médico Psiquiatra, Professor Adjunto no Departamento de Psiquiatria e Neurologia da UFMG, Doutor em Psiquiatria pela Universidade de Heidelberg.

Marco Aurélio Lana Peixoto é Médico Neurologista, Professor Adjunto nos Departamentos de Psiquiatria e Neurologia e de Oftalmologia da UFMG. Coordenador do Centro de Investigação em Esclerose Múltipla (CIEM Minas - UFMG), Doutor em Oftalmologia pela UFMG. 